\title{
Derrame pericárdico loculado en paciente con cáncer avanzado
}

\author{
Mireia Codina Prat* \\ Silvia Cisneros Carpio** \\ Cristina Asla Ormaza* \\ Lara Ruiz Gómez*

\section{Recibido: 23/06/2020 \\ Aceptado: 05/10/2020 \\ En línea: 31/12/2020}

Garazi Ramírez-Escudero Ugalde*

* Unidad de Imagen Cardíaca. Departamento de Cardiología. Hospital Universitario de Basurto. Bilbao. España

** Unidad de Tórax. Departamento de Radiología. Hospital Universitario de Basurto. Bilbao. España

\section{Correspondencia}

Mireia Codina Prat

mireiacodina7@gmail.com

Citar como: Codina Prat M, Cisneros Carpio S, Ramírez-Escudero Ugalde G, Asla Ormaza C, Ruiz Gómez L. Derrame pericárdico loculado en paciente con cáncer avanzado. Rev Ecocar Pract (RETIC). 2020 (Dic); 3 (3): 19-21. doi: 10.37615/retic.v3n3a9.

Cite this as: Codina Prat M, Cisneros Carpio S, Ramírez-Escudero Ugalde G, Asla Ormaza C, Ruiz Gómez L. Loculated pericardial effusion in a patient with advanced cancer. Rev Ecocar Pract (RETIC). 2020 (Dic); 3 (3): 19-21. doi: 10.37615/retic.v3n3a9.

\section{Palabras clave}

\section{$\triangleright$ Pericardio}

$\triangleright$ Derrame pericárdico

$\triangleright$ Imagen multimodal

$\triangleright$ TC cardíaca

\section{Keywords}

$\triangleright$ Pericardium

$\triangleright$ Pericardial effusion

$\triangleright$ Multimodality imaging

$\triangleright$ Cardiac CT

\begin{abstract}
RESUMEN
Más del 50\% de los derrames pericárdicos en pacientes oncológicos son debidos a otro proceso no maligno y por tanto es fundamental establecer la causa por sus connotaciones pronósticas y terapéuticas. La ecocardiografía transtorácica es la técnica de elección para la valoración del pericardio, pero en ocasiones la imagen multimodal puede ayudar. La tomografía computarizada (TC) ha surgido como una alternativa para determinar la cantidad y distribución del líquido y caracterizar los engrosamientos, masas y el propio líquido pericárdico, por lo que aporta información útil para determinar su etiología y guiar su drenaje. A través de un caso clínico, repasaremos brevemente la utilidad de la TC en la enfermedad pericárdica.
\end{abstract}

ABSTRACT
More than $50 \%$ of pericardial effusions in cancer patients are caused by a non-malignant process. Transthoracic
echocardiography is the first line technique for the evaluation of pericardial disease, but sometimes multimodality
imaging can help. CT have emerged as an alternative to determine the amount and distribution of the fluid and to
characterize pericardial anatomy, masses or the pericardial fluid itself providing useful information to determine
the aetiology of the effusion and to guide the management. In this clinical case we will briefly review the usefulness
ofCT in pericardial disease.

\section{Presentación del caso}

Mujer de 72 años, antigua fumadora con dislipidemia, con un adenocarcinoma de íleon que se diagnosticó en 2017 y fue tratado con quimioterapia (capecitabina y oxaliplatino) y que en la actualidad estaba en estadio IV. Debido a la mala tolerancia al tratamiento oncológico y de acuerdo con la voluntad de la paciente, se había decidido el manejo con tratamiento paliativo.

La paciente consultó por disnea progresiva hasta hasta hacerse de pequeños esfuerzos de semanas de evolucion. A la exploración física, destacaba taquicardia (110 lpm), ingurgitación yugular y tonos cardíacos apagados. El electrocardiograma (ECG) mostró taquicardia sinusal y complejos QRS de bajo voltaje. En la radiografía de tórax se observaba aumento del tamaño de la silueta cardíaca y derrame pleural bilateral, mayor en el lado izquierdo (Figura 1). Fue derivada a nuestro laboratorio para descartar taponamiento cardíaco. Se realizó ecocardiografía transtorácica (ETT) en la que se apreció un derrame pericárdico severo de contenido muy denso y un pericardio de aspecto muy engro- sado (Vídeo 1). El derrame pericárdico estaba loculado y aparentemente era más importante sobre la cara anterior del corazón (Vídeo 2). La ETT objetivó a su vez cambios respiro-fásicos marcadamente aumentados en el flujo transmitral, con variaciones del tamaño de la onda E superiores al 25\%, dilatación de la vena cava inferior (Figura 2) y colapso diastólico del ventrículo derecho (Vídeo 3) que eran compatibles con taponamiento cardíaco.

Para entender mejor la anatomía y contenido del derrame se realizó una TC de tórax que mostró el derrame loculado de localización anterior junto con engrosamientos nodulares groseros del pericardio que captaban contraste (Figura 3). Tanto el derrame pericárdico como los engrosamientos nodulares presentaban valores de atenuación altos (Figura 4). Los hallazgos sugerían, como etiologías más probables, enfermedad pericárdica maligna o infección. En nuestra paciente, la historia clínica sugería enfermedad pericárdica maligna y, tras una valoración multidisciplinar, se decidió realizar una ventana pericárdica paliativa. Se obtuvo un líquido hemático cuya citología mostró células atípicas compatibles con carcinoma. Debido a la tabicación del derrame, la ventana pericárdica consiguió sólo un drenaje parcial del líquido, pero que fue 
suficiente para mejorar la sintomatología de la paciente y que desaparecieran los datos de compromiso hemodinámico. La paciente falleció debido a progresión tumoral pocos meses tras el procedimiento.

\section{Estudio por imagen}

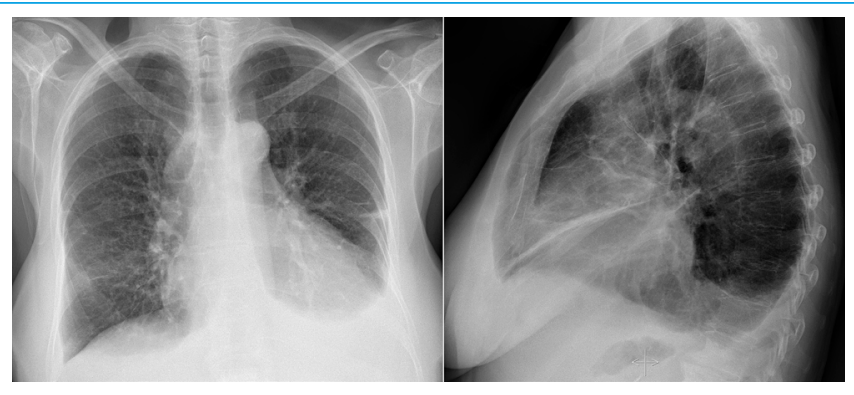

Figura 1. Radiografía de tórax. Se observa aumento del tamaño de la silueta cardíaca y derrame pleural bilateral, mayor en el lado izquierdo

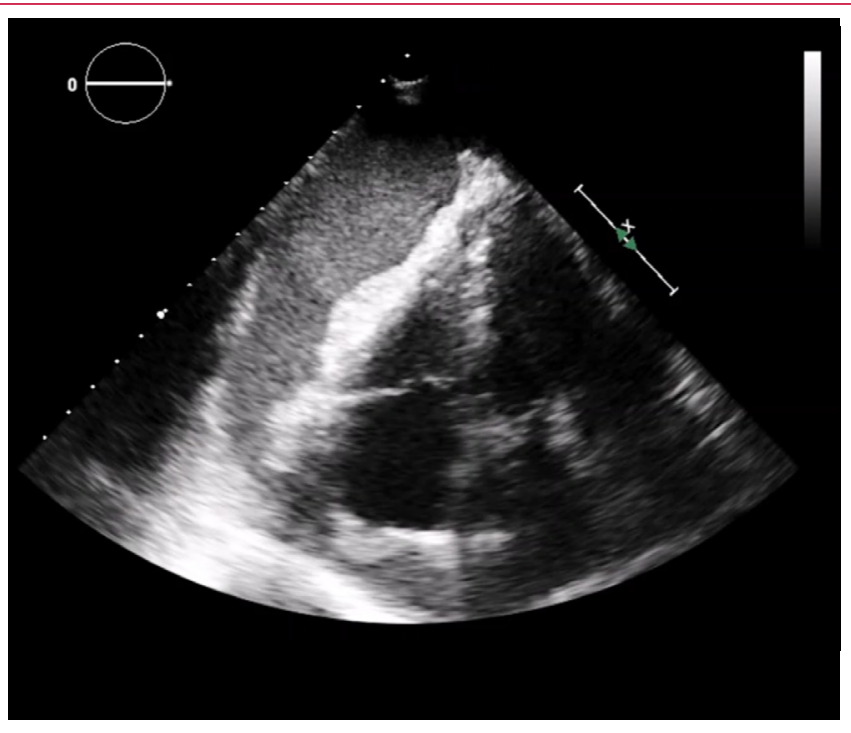

Vídeo 1. ETT. Vista apical focalizada en el ventrículo derecho donde se aprecia severo derrame pericárdico con contenido muy denso y marcado engrosamiento pericárdico

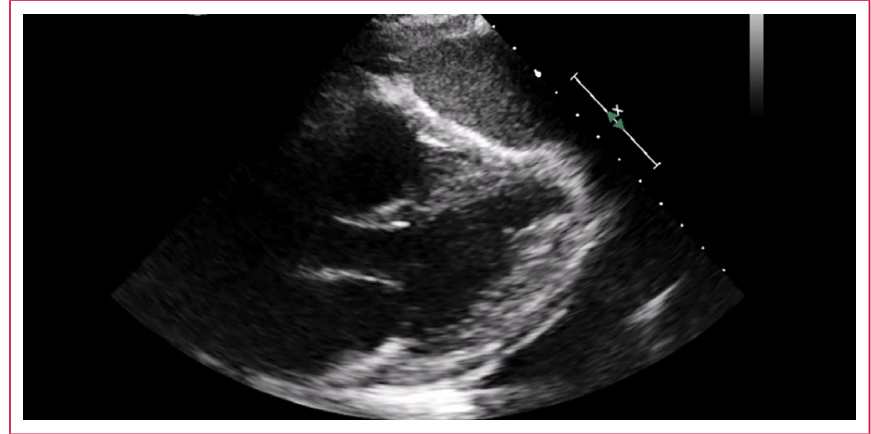

Vídeo 2. ETT. Plano paraesternal donde se comprueba la loculación y localización anterior del derrame

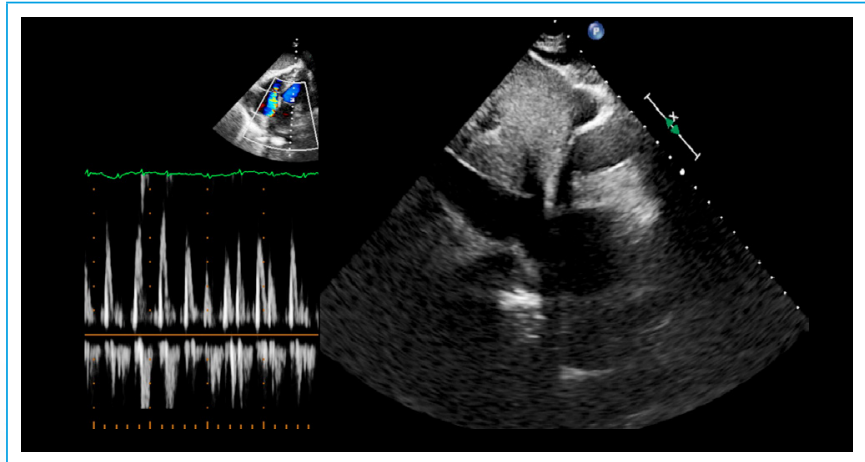

Figura 2. ETT. Cambios respiro-fásicos exagerados del flujo transmitral con variación del tamaño de la onda E superior al 25\% (izquierda) y dilatación de vena cava inferior (derecha)

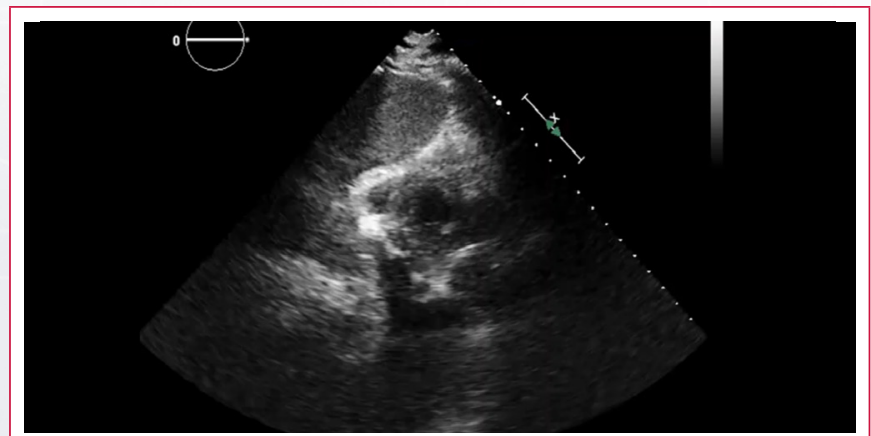

Vídeo 3. . ETT. Plano paraesternal eje corto en el que se aprecia colapso diastólico de VD

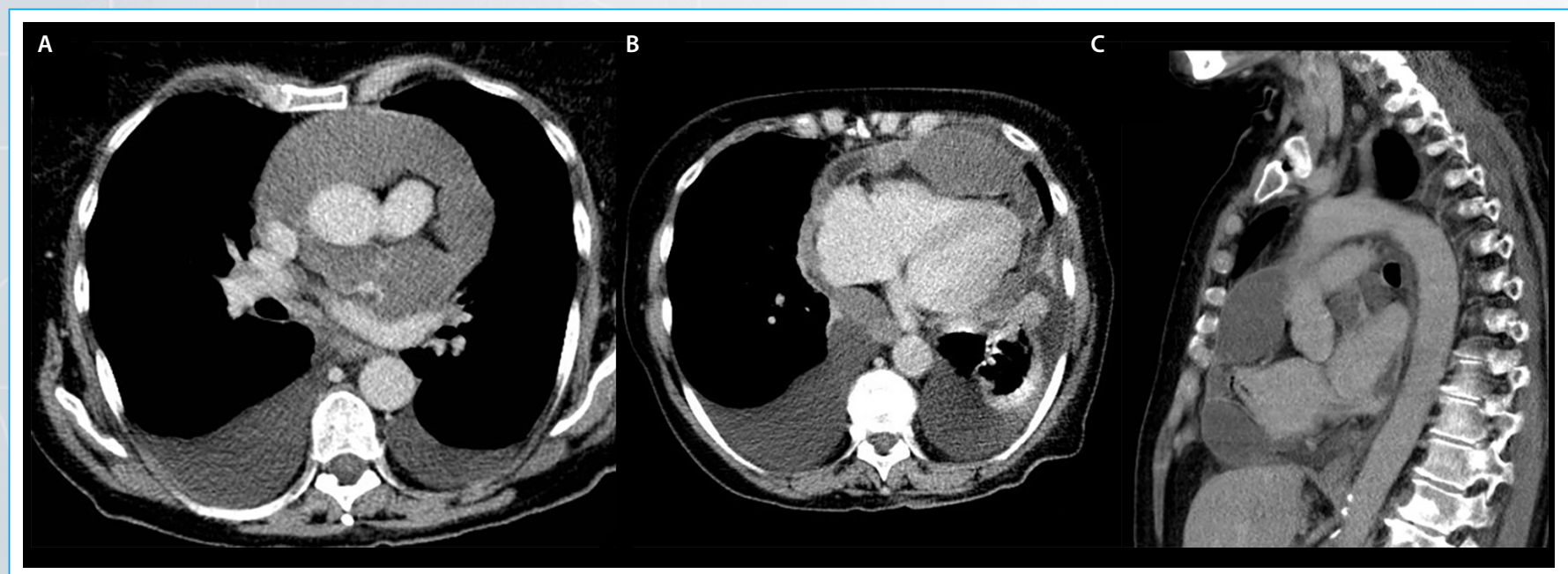

Figura 3. TC. Derrame pericárdico loculado de distribución anterior, engrosamiento pericárdico con engrosamientos nodulares con tenue captación de contraste 


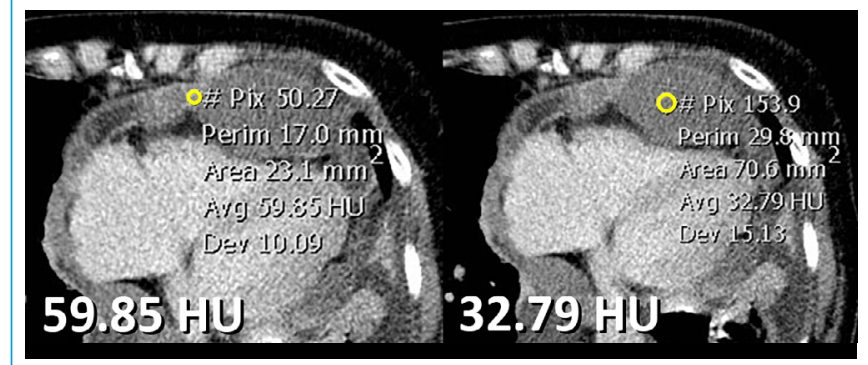

Figura 4. TC. Densidad del engrosamiento nodular $(60 \mathrm{UH})$ y del líquido pericárdico medidas por TC (33 UH)

\section{Discusión}

Los derrames pericárdicos en los pacientes oncológicos pueden ser debidos a diferentes etiologías: maligna (primaria o secundaria a metástasis); infecciosa (virus, bacterias y fúngicas, oportunistas o no oportunistas) debido a las frecuentes visitas al hospital de los paciente y a su inmunosupresión; secundaria al propio tratamiento antitumoral (radioterapia, tratamientos sistémicos como antraciclinas, mostazas nitrogenadas, inhibidores de las proteincinasas...); o secundaria a otras muchas causas, como idiopática, autoinmune, insuficiencia renal o cardíaca, traumatismos. .

Aunque un derrame pericárdico importante acompañado de taponamiento cardíaco sin signos inflamatorios en un paciente oncológico debe hacer descartar la presencia de un derrame tumoral(1), en realidad, más del $50 \%$ de los derrames en pacientes con cáncer son debidos a otro proceso no maligno, por lo que es fundamental establecer la causa subyacente para iniciar el tratamiento apropiado y no asumir automáticamente que se debe a la neoplasia. La ecocardiografía es la técnica de primera línea para la valoración de la enfermedad pericárdica, pero algunas ocasiones no es suficiente para determinar la causa y anatomía del derrame pericárdico y otras técnicas como TC o resonancia magnética (RM) pueden ayudar ${ }^{(2)}$.

La TC ha surgido como una alternativa para la valoración morfológica del derrame pericárdico ya que permite valorar la cantidad y distribución del líquido, lo que puede ser útil para guiar el drenaje, y caracterizar tanto los engrosamientos como las masas y el líquido pericárdico. Es especialmente útil cuando hay calcificaciones, ya que permite valorar su localización y la posible extensión al miocardio(3,4).

La escala de Unidades Hounsfield (UH) es una escala cuantitativa utilizada en los estudios de TC para describir los diferentes niveles de radiodensidad de los tejidos humanos. En el derrame pericárdico, los valores de atenuación radiológica pueden ser útiles tanto para la caracterización tisular como para la caracterización del líquido pericárdico. El punto de corte que se utiliza para diferenciar derrames simples o serosos de derrames hemáticos o exudativos es de $30 \mathrm{UH}$. Los derrames con valores de atenuación altos, superiores a los del agua, son sugestivos de infección, contenido hemorrágico, hipotiroidismo o malignidad. Los valores de atenuación muy bajos se han descrito en el quilopericardio. Tanto TC como RM permiten una valoración precisa del grosor y composición de las capas pericárdicas, diferenciando los derrames simples de los derrames o pericarditis inflamatorias o de las enfermedades pericárdicas malignas ${ }^{(5)}$.

Las imágenes funcionales en tiempo real de la TC sincronizada con ECG permiten detectar taponamiento y/o constricción pericárdica, pero su uso se reserva para casos de mala ventana ecocardiográfica o contraindicación para RM debido a la necesidad de exposición a mayor radiación y el uso de contraste.
Por otra parte, las metástasis en el corazón y pericardio se descubren en autopsia en un 10-12\% de los pacientes con tumores malignos. El tumor primario que afecta al pericardio más frecuentemente es el de pulmón, seguido por el de mama, el melanoma y el linfoma. Las metástasis del cáncer colorrectal al pericardio son infrecuentes, se han descrito escasos casos y suelen indicar un estadio terminal.

El derrame pericárdico maligno puede ser resultado de la diseminación del tumor al pericardio visceral que aumenta la producción de líquido o debido a la acumulación de líquido por la obstrucción del flujo venoso y/o linfático. Los derrames pericárdicos malignos se asocian a tasas de supervivencia inferiores. En la valoración por TC, los derrames pericárdicos malignos tienen altos valores de atenuación asociados a engrosamientos nodulares pericárdicos con atenuación aumentada que pueden o no captar contraste.

\section{Conclusión}

El derrame pericárdico es un problema relativamente frecuente en pacientes oncológicos que puede ser producido por una gama muy amplia de causas. En estos pacientes es fundamental establecer la causa del derrame y no asumir automáticamente que se debe a la neoplasia, ya que el impacto en el pronóstico es muy diferente. La ecocardiografía es la técnica de valoración inicial, aunque en ocasiones puede ser necesario valorar de forma más precisa el derrame con TC. Los derrames pericárdicos malignos tienen altos valores de atenuación y suelen estar asociados a engrosamientos nodulares pericárdicos con atenuación aumentada, que pueden o no captar contraste. Radiológicamente, es difícil diferenciar un derrame pericárdico maligno de uno de etiología infecciosa, en cuyo caso la clínica, citología y valoración por TC del resto de estructuras corporales es fundamental.

\section{Ideas para recordar}

- En pacientes oncológicos en los que aparece un derrame pericárdico importante que cursa con taponamiento sin signos inflamatorios se debe descartar que el derrame sea tumoral.

- Sin embargo, al estudiar un derrame o pericarditis en un paciente con cáncer es importante investigar la causa subyacente y no asumir automáticamente que su causa es neoplásica.

- La imagen multimodal ofrece ventajas en la valoración de las enfermedades del pericardio, sobre todo en enfermedad metastásica. La TC se puede utilizar como alternativa a la RM para caracterizar el pericardio, sobre todo si hay calcificaciones.

\section{Bibliografía}

1. Sagristà-Sauleda J, Mercé J, Permanyer-Miralda G, Soler Soler J. Clinical clues to the causes of large pericardial effusions. Am J Med 2000; 109: 95-101.

2. Bogaert J, Francone M. Pericardial disease. Value of $C T$ and MR imaging. Radiology 2013; 267 (2): 340-356.

3. Ünal E, Karcaaltincaba M, Akpinar E, Ariyurek OM. The imaging appearances of various pericardial disorders. Insights into Imaging 2019; 10 (1).

4. Rajiah P, Kanne JP. Computed tomography of the pericardium and pericardial disease. Journal of Cardiovascular Computed Tomography (Internet) 2010; 4 (1): 3-18.

5. Ala CK, Klein AL, Moslehi JJ. Cancer treatment-associated pericardial disease: epidemiology, clinical presentation, diagnosis, and management (Internet). Current Cardiology Reports. Springer 2019; 21: 1-9. Disponible en: https://link.springer.com/article/10.1007/s11886-019-1225-6 